\title{
Pulmonary Agenesis and Associated Pulmonary Hypertension: A Case Report and Review on Variability, Therapy, and Outcome
}

\author{
Oliver Muensterer ${ }^{1}$ Rosanna Abellar ${ }^{2}$ David Otterburn ${ }^{3}$ Rajamma Mathew ${ }^{4}$
}

${ }^{1}$ Department of Pediatric Surgery, University Medicine Mainz, Johannes Gutenberg University, Mainz, Germany

2 Department of Pathology, Columbia University, New York, New York, United States

${ }^{3}$ Department of Plastic and Reconstructive Surgery, Weill Cornell Medical College, New York, New York, United States

${ }^{4}$ Department of Pediatric Cardiology, New York Medical College, Valhalla, New York, New York, United States

\author{
Address for correspondence Oliver Muensterer, Deparment of \\ Pediatric Surgery, University Hospital Mainz, Johannes Gutenberg \\ University, Mainz, Germany, Langenbeckstrasse 1, 55101 Mainz, \\ Germany (e-mail: oliver.muensterer@unimedizin-mainz.de).
}

Eur J Pediatr Surg Rep 2015;3:33-39.

\author{
Abstract \\ Keywords \\ - pulmonary agenesis \\ - pulmonary \\ hypertension \\ - tissue expander \\ - thoracoscopy
}

Pulmonary agenesis is a rare congenital disorder with large variability in presentation and prognosis. We describe a full-term infant born with right-sided pulmonary agenesis who underwent thoracoscopic placement of a tissue expander. He ultimately died of pulmonary hypertension. Immunohistology showed intimal hyperplasia without the loss of endothelial caveolin-1 expression. A literature review revealed that while some of these patients have favorable outcome, many succumb despite therapy.

\section{Introduction}

Pulmonary agenesis is a rare congenital defect, often associated with a spectrum of other malformations, and syndromes. ${ }^{1}$ About one-fifth of patients also present with varying degrees of pulmonary hypertension (PH). ${ }^{2,3}$ Associated comorbidities negatively impact on the prognosis. Due to the absence of one lung, the mediastinal shift toward the affected side impacts vessels and airways similarly to what is observed in postpneumonectomy syndrome. ${ }^{4}$ These changes by themselves may cause airway compromise, ${ }^{5}$ or modify hemodynamics, leading to secondary change including pulmonary hypertension. To treat the mechanical sequelae of pulmonary agenesis, pediatric surgeons in select centers have placed tissue expanders into the ipsilateral chest in an attempt to shift the mediastinum into its anatomic location. ${ }^{6}$ Reports on this intervention are sporadic, with varying outcomes.

We describe the case of a male infant born with right pulmonary agenesis who underwent thoracoscopic placement of a tissue expander. Despite the intervention, his pulmonary hypertension worsened, and he eventually succumbed to pneumonia and sepsis. We performed a detailed analysis of clinical course, as well as the pulmonary tissues obtained at autopsy. In addition, we examined the expression of caveolin-1, von Willebrand factor (vWF) and smooth muscle- $\alpha$-actin (SM$\alpha$-actin). A systematic literature review was performed for similar published cases using the search terms "pulmonary agenesis," "pulmonary agenesis and pulmonary hypertension," "pulmonary agenesis and right ventricular failure," as well as "pulmonary agenesis and tissue expander" in PubMed. Titles were scanned for relevance. Total published cases were estimated and associated malformations were compiled. Cases with associated pulmonary hypertension were evaluated in terms of demographics, interventions, and outcome.

\section{Case Report}

\section{Clinical History}

A male infant was born at full term with a prenatal diagnosis of right pulmonary agenesis by ultrasound. After delivery, he was
License terms

Stuttgart · New York

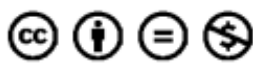

August 6, 2014

accepted after revision

October 2, 2014

published online

January 8, 2015

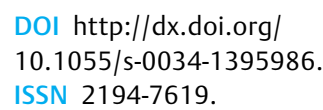



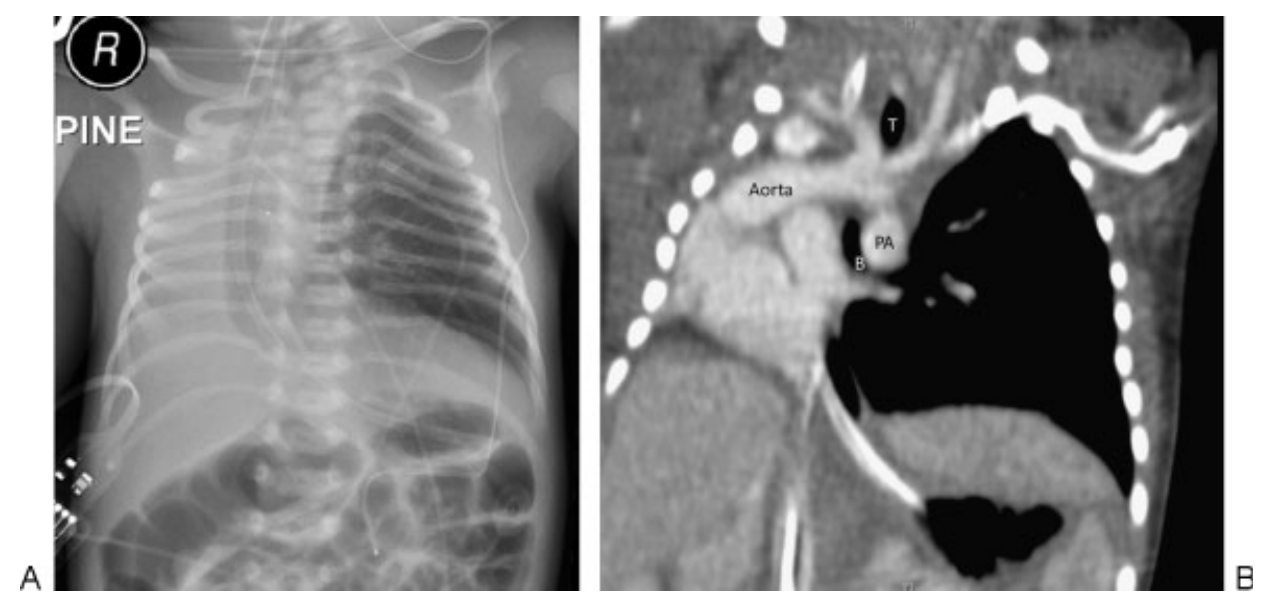

Fig. 1 Postnatal chest radiograph (A) showing right pulmonary agenesis, and mediastinal shift toward the right. Frontal reconstruction of the chest computed tomography shows the airway (T-trachea, B-bronchus) coursing around the pulmonary artery (PA) into the emphysematous unilateral left lung (B).

intubated and ventilated on low ventilatory settings, subsequently weaned to continuous positive airway pressure (CPAP) over the following 4 days. Pulmonary agenesis was confirmed by chest radiograph ( - Fig. 1A). Nasopharyngeal CPAP was tolerated for 48 hours; then he required reintubation for progressive tachypnea and hypercarbia. Nutrition was administered intravenously initially, then via a nasogastric tube. Echocardiography showed an interrupted inferior vena cava with azygos continuation, varying dilatation of the main pulmonary artery, and pulmonary hypertension. On computed tomography, there was mediastinal shift to the right. The trachea and narrowed left bronchus coursed around the pulmonary artery and toward the single left lung ( - Fig. 1B). Emphysematous changes were seen in the left lung.

\section{Surgery}

At the age of 3 months, in an effort to restore the mediastinum to the midline and thereby potentially improve the airway narrowing, the plan was made to insert a $50 \mathrm{~mL}$ rectangular tissue expander with a remote port into the right hemithorax and gradually fill it with saline. Preoperative bronchoscopy was performed which showed significant lower airway narrowing ( - Fig. 2A). The patient was positioned 45 degrees left lateral decubitus. Placement of the tissue expander was performed thoracoscopically through two incisions $(10-\mathrm{mm}$ incision at seventh intercostal space midaxillary line and 5-mm incision at fourth intercostal space anterior axillary line). Upon entering the chest through the lower incision, there was no obvious pleural space. A 14F Foley catheter was blindly and gently advanced through the incision and the balloon slowly inflated to $8 \mathrm{~mL}$. This created a space into which the endoscope could be advanced. The same technique was initially used at the other access site, and then the space was bluntly augmented using a cotton swab or endoscopic Kittner dissector. Once the new right hemithoracic space was created, the tissue expander was introduced into the chest. A test filling of $30 \mathrm{~mL}$ was performed, and intraoperative echocardiography confirmed that there was no compromise to hemodynamics. Repeat bronchoscopy showed improved lower airway caliber ( - Fig. 2 B).The filling port was then secured in the subcutaneous space and the skin closed.

\section{Postoperative Hospital Course}

The expander was filled with aliquots of 2 to $5 \mathrm{~mL}$ of saline daily, up to a total maximal volume of $45 \mathrm{~mL}$. Over the
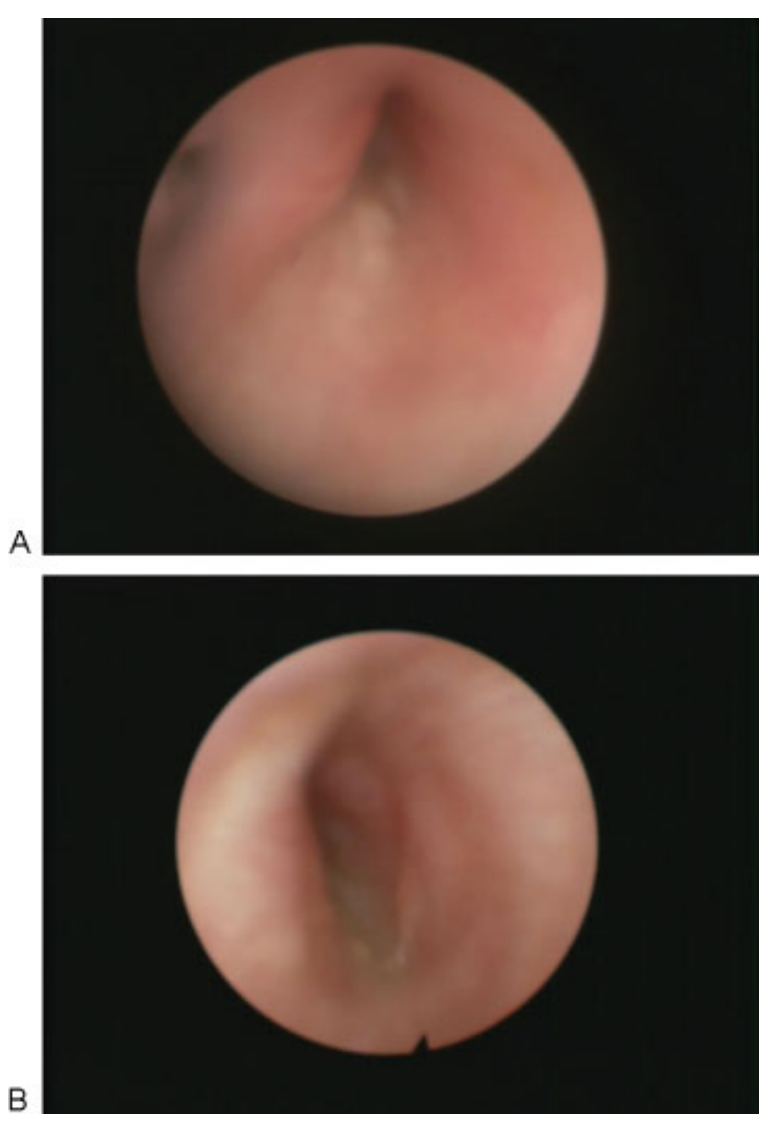

Fig. 2 Preoperative bronchoscopy showed significant, pulsatile narrowing of the lower airway, most likely due to the large adjacent pulmonary artery (PA) (A). After placing and inflating the tissue expander in the right chest, the narrowing seemed to have improved (B). 
following weeks, the patient's ventilatory needs fluctuated. At times, weaning seemed possible, but at other times greater ventilatory support was required. Follow-up echocardiographs suggested varying degrees of pulmonary hypertension, and a pulmonary artery that slowly increased in size. He required cardiopulmonary resuscitation twice, and was changed to high-frequency oscillator ventilation. The pulmonary hypertension was treated with nitric oxide (NO) at $20 \mathrm{ppm}$ in the inhaled gas mix, a Fio2 of 90\%, and milrinone for severe right ventricular dysfunction. Cardiac catheterization performed 2 weeks after the start of $\mathrm{NO}$ and milrinone therapy revealed the absence of intracardiac shunting, right arterial pressure of $19 \mathrm{~mm} \mathrm{Hg}$, right ventricular pressures of $95 / 28 \mathrm{~mm} \mathrm{Hg}$, and pulmonary artery pressures of 101/59 (mean 76) $\mathrm{mm} \mathrm{Hg}$ at baseline off NO. He did not respond to $80 \mathrm{ppm} \mathrm{NO}$ in the catheterization laboratory. Filling of the tissue expander under controlled conditions in the catheterization laboratory did not compromise hemodynamics or worsen pulmonary hypertension. There was a large left pulmonary artery without obstruction, but with abnormal distal arborization by angiography suggesting chronic pulmonary hypertension. Eventually, he developed pneumonia, sepsis, and expired at 5 months of age, 3 days after the cardiac catheterization.

\section{Pathology}

Pertinent findings on autopsy were a 5,180 g male infant with unilateral pulmonary agenesis and absence of the right bronchus. The left unilobar lung weighed $130 \mathrm{~g}$. The diameter of the trachea was $1.2 \mathrm{~cm}$, the left bronchus $0.6 \mathrm{~cm}$, there was absence of a carina. Marked cardiomegaly (heart weight $58 \mathrm{~g}$, normal $35 \pm 5 \mathrm{~g}$ ) was found, with a patent foramen ovale and subendocardial acute myocardial infarction of the right ventricle with reperfusion injury.

\section{Histology and Immunofluorescence}

Formaldehyde preserved lung tissue was processed for paraffin blocks. Approximately 5 to $6 \mu \mathrm{m}$ sections were stained with hematoxylin \& eosin (H\&E) and elastic Van Gieson (EVG) stain to examine the lung histology. The pulmonary arteries showed intimal hyperplasia and medial hypertrophy (-Fig. 3), Histopathologic changes were consistent with pulmonary hypertension (Heath-Edwards II/VI ${ }^{7}$ ). In addition, using double immunofluorescence technique, we examined the expression of caveolin- $1, \mathrm{vWF}$, and SM- $\alpha$-actin (a marker for smooth muscle cells). The expression of endothelial caveolin-1 (-Fig. 4A) was well preserved, indicating that there was no disruption of the endothelial cells. In a few arteries there was intimal thickening without loss of endothelial caveolin-1 ( - Fig. 4A, bottom lanes), consistent with the H\&E and EVG stains. The expression of VWF was also well preserved (data not shown). - Fig. 4B shows several small vessels expressing endothelial caveolin-1 with the lack of muscularization.

\section{Results of Literature Review}

The search term "pulmonary agenesis" yielded 288 articles in PubMed. Since 1946, the year in which a landmark

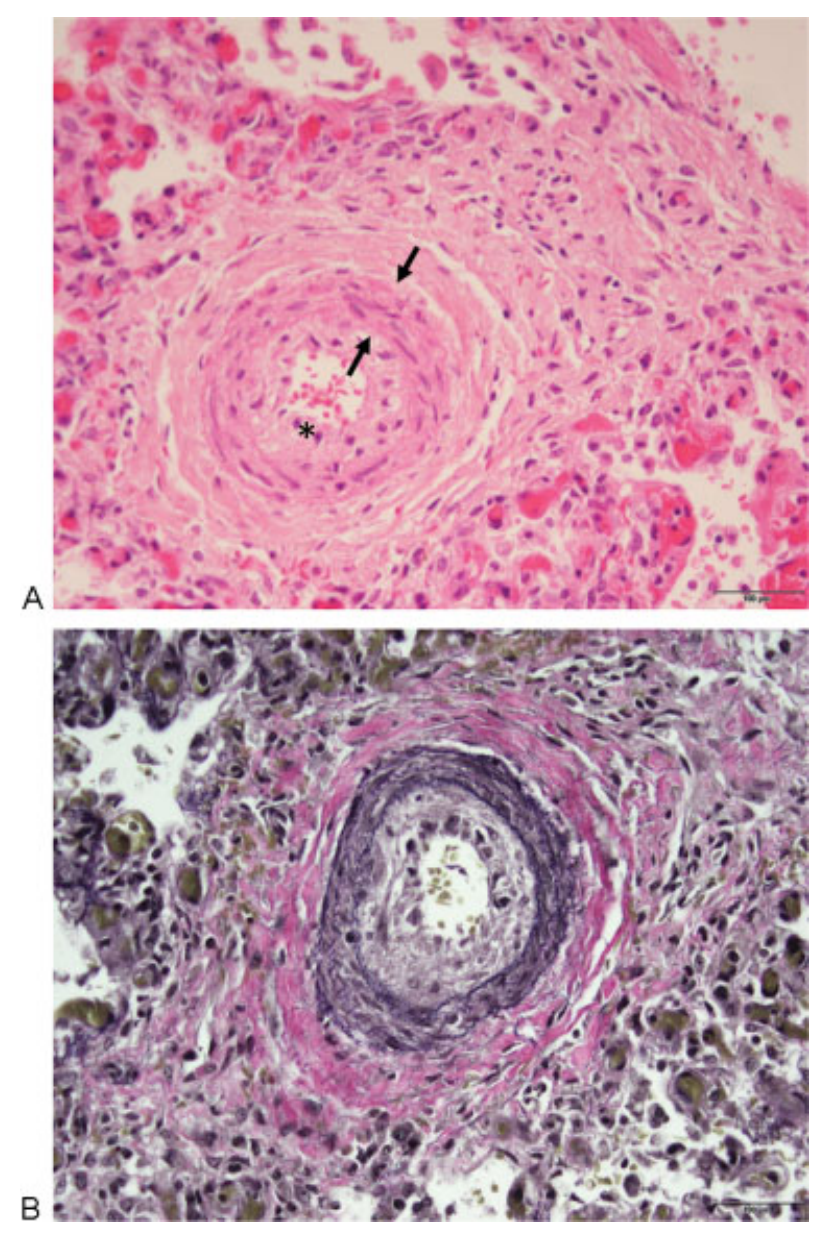

Fig. 3 Hematoxylin and eosin stain (A) of a typical arteriole showing increased cellularity in the intima $\left({ }^{*}\right)$ and thickening of the tunica media (arrows). A corresponding elastic-van gieson stain (B) shows intimal hyperplasia and medial hypertrophy of the tunica media in a small artery (magnification $\times 20$ ).

review appeared summarizing 50 previously published cases, ${ }^{8}$ at least 300 cases have been described in the literature. The most common associated anomalies were structural cardiac disease, esophageal atresia with or without tracheoesophageal fistula, diaphragmatic defects, and limb anomalies. Other published associated anomalies are listed in -Table 1.

Combining "pulmonary agenesis and pulmonary hypertension" generated 10 results. The sequence "pulmonary agenesis and right ventricular failure" returned another five articles, "pulmonary agenesis and tissue expander" an additional four. These articles were screened for patients who had pulmonary hypertension associated with their pulmonary agenesis, and a total of 20 affected individuals were found in 15 publications spanning from 1978 to 2011, most being single case reports. Presenting patient ages ranged from 1 day to 51 years (median 70 days), gender, and laterality was equally distributed. There were $12(60 \%)$ long-term survivors in the published collective. The most common surgeries were repair of the associated structural heart disease $(n=5)$, and tracheoplasty on extracorporeal membrane oxygenation (ECMO) $(n=2)$. 


\section{cav-1}
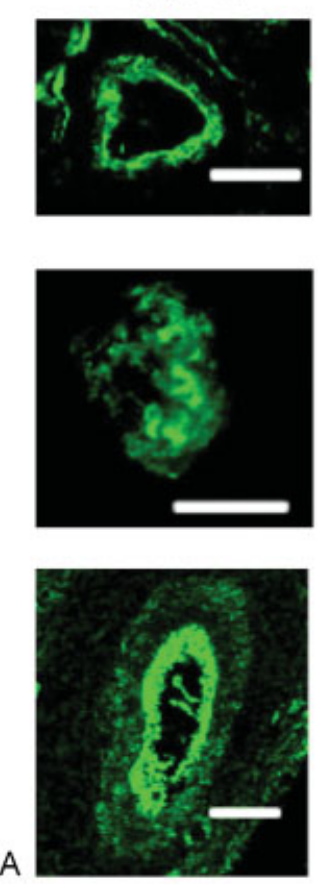

A

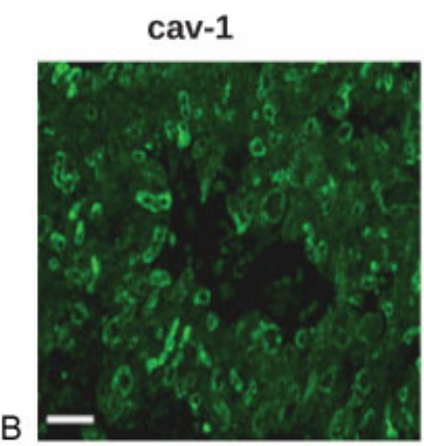

$\alpha$-actin
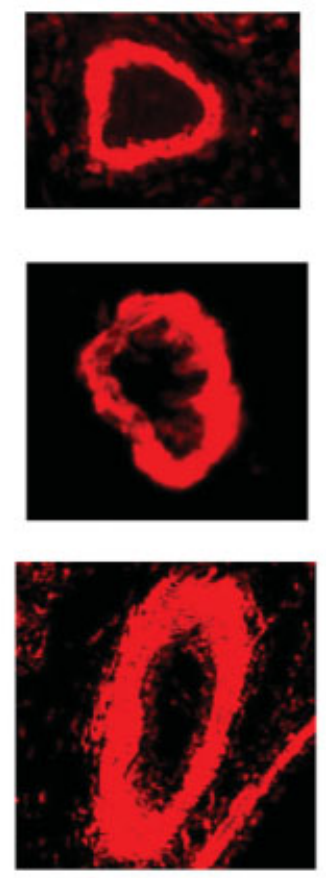

$\alpha$-actin

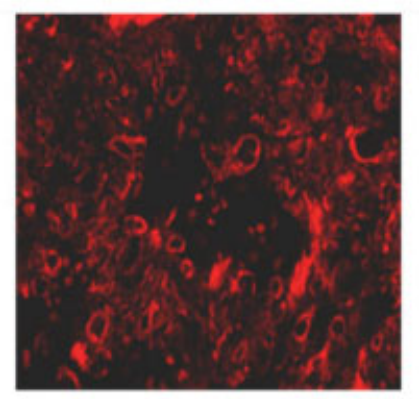

merge
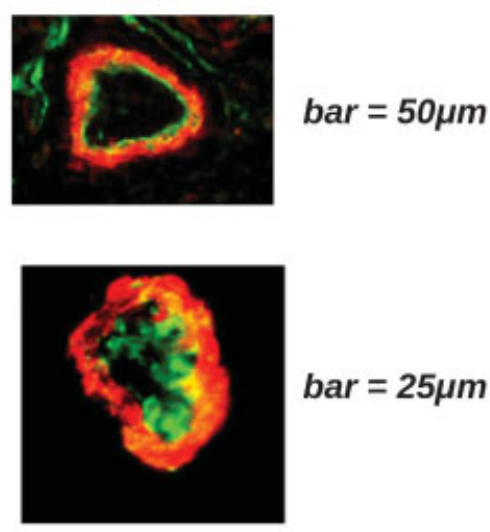

bar $=25 \mu m$

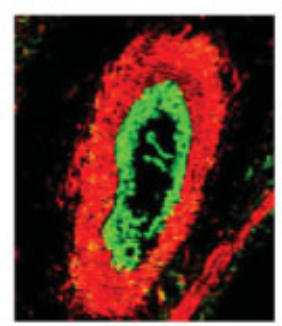

merge

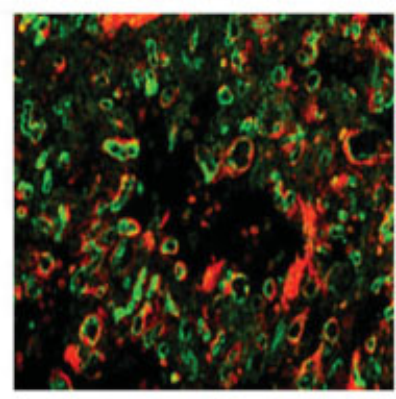

bar $=50 \mu \mathrm{m}$

Fig. 4 Expression of caveolin-1 (green) and smooth muscle $\alpha$-actin (red) in pulmonary arteries (A). The top panel shows thin intimal layer with well-preserved endothelial caveolin-1. The lower panels show a thickened intimal layer with well-preserved endothelial caveolin-1 and without endothelial disruption. In (B), small vessels with endothelial caveolin-1 (green) expression are seen. Several of these vessels lack the expression of smooth muscle $\alpha$-actin (red).

There were three published patients in whom a tissue expander was placed by open thoracotomy. ${ }^{4,6,9,10}$ Bittigau et al and Kalache et al describe the same patient. The ages were 3 months, 5 months, and 1 year, with initial filling volumes of 40,80 , and $140 \mathrm{~mL}$, respectively. Only one of the patients ${ }^{10}$ had pulmonary hypertension, which resolved postoperatively. However, the patient underwent transient tracheostomy for tracheal narrowing. All reported cases treated with the tissue expander were males, and all had right-sided pulmonary agenesis.

\section{Discussion}

Pulmonary agenesis is exceedingly rare, and the vast majority of pediatric surgeons' cumulative experience will barely surpass the single digits. The incidence is estimated at around 1 in 15,000 pregnancies. ${ }^{11}$ It was first described by De Pozze in 1673 as an incidental finding during an autopsy of an adult woman. ${ }^{8}$ Patients frequently have other anomalies, including congenital heart disease. The long-term survival into adulthood is estimated at around 50\% for agenesis of the left lung, and $30 \%$ in patients with the right lung affected. ${ }^{12}$

The clinical presentation of pulmonary agenesis is extremely variable. The author (O. M.) has personally taken care of four patients with pulmonary agenesis during training and practice, all with markedly different outcome. Besides the described patient in this report, one patient presented with airway narrowing, stridor, but no significant pulmonary hypertension at birth. The patient underwent tracheoplasty and became asymptomatic. Another neonate was referred in immediate postnatal respiratory distress. The initial radiograph showed right-sided pulmonary agenesis, and persistent hypoxia prompted ECMO. The pulmonary hypertension never resolved and the patient eventually died. Finally, a 
Pulmonary Agenesis and Associated Pulmonary Hypertension Muensterer et al. 37

Table 1 Associated anomalies published in patients with pulmonary agenesis

\begin{tabular}{|c|c|}
\hline Category & Types of anomalies \\
\hline \multirow[t]{9}{*}{ Head and Neck } & - Microtia \\
\hline & - Other ear malformations \\
\hline & - Microphthalmia \\
\hline & - Asymmetric crying facies \\
\hline & - Colloidal goiter \\
\hline & - Cleft lip, palate \\
\hline & - Maxillary hypoplasia \\
\hline & - Mandibular hypoplasia \\
\hline & - Velocardiofacial syndrome \\
\hline \multirow[t]{24}{*}{ Cardiothoracic } & - Sternal defect \\
\hline & - Azygos vein duplication \\
\hline & - Atrial septal defect \\
\hline & - Patent foramen ovale \\
\hline & - Common atrium \\
\hline & - Ventricular septal defect \\
\hline & $\begin{array}{l}\text { - Common origin of } \\
\text { both coronary arteries }\end{array}$ \\
\hline & $\begin{array}{l}\text { - Total anomalous } \\
\text { pulmonary venous return }\end{array}$ \\
\hline & - Tetralogy of Fallot \\
\hline & - Interruption of aortic arch \\
\hline & - Patent ductus arteriosus \\
\hline & - Hypoplastic pulmonary artery \\
\hline & - Vascular ring \\
\hline & - Vascular sling \\
\hline & - Tricuspid atresia \\
\hline & - Bronchoesophageal fistula \\
\hline & - Tracheal stenosis \\
\hline & - Tracheal cartilaginous rings \\
\hline & - Hiatal hernia \\
\hline & - Diaphragmatic hernia \\
\hline & - Laryngoesophageal cleft \\
\hline & - Esophageal lung \\
\hline & - Pectus excavatum \\
\hline & - Rib anomalies \\
\hline \multirow[t]{6}{*}{ Limb anomalies } & - Amelia \\
\hline & - Radial ray deficit \\
\hline & - Phalanx hypoplasia \\
\hline & - Intrathoracic upper limb \\
\hline & - Polydactyly \\
\hline & - Bifid thumb \\
\hline
\end{tabular}

(Continued)
Table 1 (Continued)

\begin{tabular}{|c|c|}
\hline Category & Types of anomalies \\
\hline \multirow[t]{8}{*}{ Gastrointestinal } & $\begin{array}{l}\text { - Esophageal atresia, } \\
\text { tracheoesophageal fistula }\end{array}$ \\
\hline & - Gastroesophageal reflux \\
\hline & - Gastric duplication \\
\hline & - Pyloric stenosis \\
\hline & - Biliary atresia \\
\hline & - Duodenal atresia \\
\hline & - Meckel diverticulum \\
\hline & - Anorectal malformations \\
\hline \multirow[t]{9}{*}{ Visceral, genitourinary } & - Situs inversus \\
\hline & $\begin{array}{l}\text { - Ectopia hepatica } \\
\text { extrathoracica }\end{array}$ \\
\hline & - Exomphalos \\
\hline & - Renal agenesis \\
\hline & - Crossed renal ectopia \\
\hline & - Horseshoe kidney \\
\hline & - Vesicoureteral reflux \\
\hline & - Uterovaginal duplication \\
\hline & - Müllerian agenesis \\
\hline \multirow[t]{12}{*}{ Genetic, syndromes } & - Nandini-Nyhan association \\
\hline & - Holt-Oram syndrome \\
\hline & - Down syndrome \\
\hline & - Goldenhar syndrome \\
\hline & - First arch syndromes \\
\hline & - DiGeorge syndrome \\
\hline & - Nager acrofacial dysostosis \\
\hline & - VACTERL association \\
\hline & - Partial trisomy $21 \mathrm{q}$ \\
\hline & - Partial/complete trisomy 2p \\
\hline & - $46 \times X 2 p+$ \\
\hline & - 22q11.2 deletion \\
\hline \multirow[t]{7}{*}{ Other } & - Neuroblastoma \\
\hline & - Occipital encephalocele \\
\hline & - Spinal deformities \\
\hline & - Hemivertebrae \\
\hline & - Fused vertebrae \\
\hline & - Hydrops fetalis \\
\hline & - Arteriovenous malformations \\
\hline
\end{tabular}


3-year-old presented to the emergency room for workup of an upper respiratory infection. He was found to have right pulmonary agenesis, but was otherwise completely healthy and asymptomatic. These strikingly different presentations underline the variability of pulmonary agenesis. This variability may depend on the cross-sectional area of the pulmonary vasculature or other variables. Further experimental studies are therefore required to understand the mechanism/s underlying pulmonary agenesis and determine prognosis.

The signs and symptoms in some patients with pulmonary agenesis mimic changes seen after removal of an entire lung for other underlying disease, termed postpneumonectomy syndrome. ${ }^{13}$ The resulting mediastinal shift may lead to extrinsic tracheal compression and airway stretching, as observed in our case. It also leads to overextension and emphysematous changes in the contralateral, remaining lung. At times, vascular compression or kinking is also observed. Interestingly, due to the anatomic relationship of the airways, the esophagus, and the great vessels, right postpneumonectomy syndrome is generally considered to have worse prognosis than left, ${ }^{14,15}$ and early intervention in symptomatic patients is warranted.

Pulmonary hypertension is thought to occur in approximately $19 \%$ of patients with pulmonary agenesis. This association increases to $88 \%$ when there is associated left-to-right shunting. ${ }^{3}$ In our literature review, we found 20 reported patients with pulmonary hypertension in the setting of pulmonary agenesis, roughly in line with the above quoted proportions. Also, corroborating the referenced source, many of the reported patients had structural heart disease predisposing to shunting with increased pulmonary blood flow. Whether pulmonary hypertension in the setting of pulmonary agenesis is a primary phenomenon based on malformation of the pulmonary vasculature or a secondary phenomenon due to the anatomic shift and consequential effects on the vessels and bronchus, has not yet been defined. One interesting aspect of our case is that despite increased pulmonary artery pressure and media wall thickening, the intimal layer was not disrupted. It is important to note that there was no associated intra-cardiac shunt. This is consistent with our previous studies showing that the elevated pulmonary artery pressure alone in the absence of associated intracardiac shunt or inflammation does not disrupt the intimal layer. ${ }^{16}$ However, in our present case, there is evidence of intimal proliferation. Caveolin-1 is a major scaffolding protein of caveola, a subset of lipid rafts found on plasmalemmal membranes of a variety of cells. Caveolin-1 interacts with transducing molecules that reside in or recruited to caveolae. Caveolin-1 inhibits proliferative pathways, and modulates cell cycle and inflammation, thus, maintains vascular homeostasis. Loss of caveolin-1 is generally considered indicative of endothelial damage, and is usually progressive in $\mathrm{PH}$. As the disease progresses the loss of vWF found in Weibel-Palade bodies in endothelial cells occurs, which is indicative of extensive endothelial damage or loss. Importantly, at this stage enhanced expression of caveolin-1 in smooth muscle cells becomes pro-proliferative and worsens the disease. Conversely, loss of vWF indicates extensive endothelial damage or loss. Interestingly, the loss of caveolin-1 and vWF expression has been described in infants with $\mathrm{PH}$ and associated increased pulmonary flow or underlying inflammatory disease, but not with $\mathrm{PH}$ alone. ${ }^{16}$ The expression of endothelial caveolin-1 was well preserved in the present case, indicating that there was no disruption of the endothelial cells. However, a few arteries exhibited intimal thickening without the loss of endothelial caveolin-1. Further studies are necessary to identify the underlying mechanisms of pulmonary agenesis, vascular development, associated $\mathrm{PH}$, and intimal hyperplasia.

Pulmonary agenesis is associated with a variety of other disorders. On first glance, there seems to be no common systematic mechanism in the multitude of malformations. Mutations in the T-box genes can produce a combination of cardiopulmonary and extremity malformations (classically, for example, Holt-Oram syndrome), along with urogenital defects. ${ }^{17}$ Cardiac and laterality defects including the oculofaciocardiodental syndrome, may be due to suppression of the PITX2 gene in the embryonic lateral plate mesoderm. ${ }^{18} \mathrm{An}$ interesting observation in this context is that in most cases of pulmonary agenesis, the associated malformations seem to be ipsilateral. $^{19}$

Surgical therapy includes correction of the associated cardiac defects, particularly if these are severe or cause significant morbidity. ${ }^{20-22}$ Tracheobronchial compression has been addressed by aortopexy, ${ }^{23}$ and tracheoplasty on and off ECMO. ${ }^{5,24,25}$ Implantation of a tissue expander for pulmonary agenesis was first described in 1995 in a report from Berlin, Germany. ${ }^{6}$ Other centers that have reported this approach have been Bordeaux, France, ${ }^{10}$ and Verona, Italy. ${ }^{4}$ All of the described procedures were performed via open thoracotomy. Incidentally, our patient is the first report in which a tissue expander was implanted thoracoscopically. The procedure itself was tolerated by the patient without any problems. However, expander placement was meticulously discussed among the team, and the parents were heavily involved in the treatment decision after full disclosure of the published evidence and the potential risks. We do not know if the expander itself caused the worsening of pulmonary hypertension. Only a prospective registry of these patients can perhaps shed light on the effects of different therapeutic interventions in the future.

It is important to have the capability to perform intraoperative echocardiography during the procedure to make sure that there is no compression or compromise of the heart when the expander is filled for the first time. A very different surgical approach has been described in Kiev, Ukraine, consisting of the cephalad translocation and fixation of the ipsilateral diaphragm. This approach was performed in two patients with good long-term results. ${ }^{26}$

The published cases of tissue expander implantation may not be a complete account of how often this therapy was employed, since surgeons are less inclined to publish negative outcomes. In our case, we hesitated as well, but ultimately decided to report this patient to obtain a more complete collective experience. Judging by the many reports that have been published in the last decades, the incidence, and thereby 
the impact, may be underestimated. It would be important to pool future cases and build a registry, including the analysis of lung tissue samples from patients who expired. Going forward, we hereby would like to invite clinicians to contact us with their cases, even if the outcome was unfavorable. By joining our efforts, we hope to one day understand why some of these children are asymptomatic, and how symptomatic patients are optimally treated.

\section{References}

1 Kayemba-Kay's S, Couvrat-Carcauzon V, Goua V, et al. Unilateral pulmonary agenesis: a report of four cases, two diagnosed antenatally and literature review. Pediatr Pulmonol 2014;49(3): E96-E102

2 Sit KF, Huang TT. Anesthesia for pulmonary agenesis with pulmonary hypertension: a case report. Ma Zui Xue Za Zhi 1987;25(1): 43-48

3 Capuani A, Del Torso S, Lincoln JC. Right lung agenesis. A case report. G Ital Cardiol 1982;12(5):381-383

4 Furia S, Biban P, Benedetti M, Terzi A, Soffiati M, Calabrò F. Postpneumonectomy-like syndrome in an infant with right lung agenesis and left main bronchus hypoplasia. Ann Thorac Surg 2009;87(5):e43-e45

5 Harrison MR, Hendren WH. A genesis of the lung complicated by vascular compression and bronchomalacia. J Pediatr Surg 1975; 10(5):813-817

6 Bittigau K, Bohm J, Kursawe R, Nowotny T, Bittigau P, Konertz W. Pulmonary agenesis in a newborn: implantation of tissue expander to prevent a mediastinal shift. Thorac Cardiovasc Surg 1995; 43(5):287-289

7 Heath D, Edwards JE. The pathology of hypertensive pulmonary vascular disease; a description of six grades of structural changes in the pulmonary arteries with special reference to congenital cardiac septal defects. Circulation 1958;18(4 Part 1):533-547

8 Field CE. Pulmonary agenesis and hypoplasia. Arch Dis Child 1946; 21:61-75

9 Kalache KD, Chaoui R, Paris S, Bollmann R. Prenatal diagnosis of right lung agenesis using color Doppler and magnetic resonance imaging. Fetal Diagn Ther 1997;12(6):360-362

10 Dobremez E, Fayon M, Vergnes P. Right pulmonary agenesis associated with remaining bronchus stenosis, an equivalent of postpneumonectomy syndrome. Treatment by insertion of tissue expander in the thoracic cavity. Pediatr Surg Int 2005;21(2): 121-122

11 Bianchi DW, Crombleholme TM, D'Alton ME. Pulmonary agenesis. In: Bianchi DW, Chrombelholme TM, D'Alton ME, Malone FD, eds.
Fetology: Diagnosis and Management of the Fetal Patient. New York, NY: McGraw-Hill; 2010:300-306

12 Schaffer AJ, Rider RV. A note on the prognosis of pulmonary agenesis and hypoplasia according to the side affected. J Thorac Surg 1957;33(3):379-382

13 Soll C, Hahnloser D, Frauenfelder T, Russi EW, Weder W, Kestenholz PB. The postpneumonectomy syndrome: clinical presentation and treatment. Eur J Cardiothorac Surg 2009;35(2):319-324

14 da Silva PS, de Oliveira R, Tamura N, Neto HM. Right pneumonectomy syndrome: a late life-threatening complication of pneumonectomy. Pediatr Emerg Care 2010;26(7):499-502

15 Amin R, Joshi V, Cox P. Right pneumonectomy syndrome presenting as a cardiac arrest in a two-year-old. Pediatr Crit Care Med 2009;10(1):e9-e11

16 Dereddy N, Huang J, Erb M, et al. Associated inflammation or increased flow-mediated shear stress, but not pressure alone, disrupts endothelial caveolin-1 in infants with pulmonary hypertension. Pulm Circ 2012;2(4):492-500

17 Bogarapu S, Bleyl SB, Calhoun A, et al. Phenotype of a patient with contiguous deletion of TBX5 and TBX3: expanding the disease spectrum. Am J Med Genet A 2014;164A(5):1304-1309

18 Tanaka K, Kato A, Angelocci C, Watanabe M, Kato Y. A potential molecular pathogenesis of cardiac/laterality defects in OculoFacio-Cardio-Dental syndrome. Dev Biol 2014;387(1):28-36

19 Cunningham ML, Mann N. Pulmonary agenesis: a predictor of ipsilateral malformations. Am J Med Genet 1997;70(4):391-398

20 Hasegawa T, Oshima Y, Maruo A, et al. Pediatric cardiothoracic surgery in patients with unilateral pulmonary agenesis or aplasia. Ann Thorac Surg 2014;97(5):1652-1658

21 Guo HW, Pan SW, Song YH, Hu SS. Repair of a ventricular septal defect in a patient with left lung agenesis. J Card Surg 2011;26(5):519-520

22 Pietrzykowski WD, Moll M, Kobielski A, Moll JJ. Left atrial approach to close the atrial septal defect type II in case of right lung agenesis with extreme dextroversion. Interact Cardiovasc Thorac Surg 2007;6(1):126-127

23 Nowotny T, Ahrens BC, Bittigau K, et al. Right-sided pulmonary aplasia: longitudinal lung function studies in two cases and comparison to results from term healthy neonates. Pediatr Pulmonol 1998;26(2):138-144

24 Backer CL, Kelle AM, Mavroudis C, Rigsby CK, Kaushal S, Holinger LD. Tracheal reconstruction in children with unilateral lung agenesis or severe hypoplasia. Ann Thorac Surg 2009;88(2):624-630, discussion 630-631

25 Hines MH, Hansell DR. Elective extracorporeal support for complex tracheal reconstruction in neonates. Ann Thorac Surg 2003; 76(1):175-178, discussion 179

26 Krivchenya DU, Rudenko EO, Lysak SV, Dubrovin AG, Khursin VN, Krivchenya TD. Lung aplasia: anatomy, history, diagnosis and surgical management. Eur J Pediatr Surg 2007;17(4):244-250 